\title{
The use of radiofrequency for hepatocellular carcinoma ablation: an update review and perspectives
}

\begin{abstract}
The World Health Organization classifies liver cancer among the five types of cancer with highest death rates in the world. Among the current methods available for the treatment of liver cancer, there is the resection of hepatic tissue and the radiofrequency ablation of the tumor. Even though resection presents the best results, only $10 \%$ to $15 \%$ of the affected patients may eligible for this procedure. On the other hand, the radiofrequency ablation encompasses a larger scope of patients and provides a non-invasive method when compared to resection. There is research with sufficient evidence to allow the transposition of this concept to new technological paradigms, which would yield a more effective ablation process, i.e.: generating enough volumetric necrosis for complete regression of the tumor, leading to a high survival rate of patients. These technological paradigms encompass aspects of operability, innovation and of theoretical framework. In terms of operability, there is the use of better imaging sources to aid the healthcare professional in the positioning of electrodes; in terms of innovation, there are new technologies such as the use of optical fiber microsensors and metallic magnetic nanoparticles to increase the efficiency of the process; in terms of theoretical framework, there is the development of more precise mathematical models that would expand the possibilities of application and increase its effectiveness. These new challenges are new possibilities that may reshape the concept and the use of radiofrequency ablation as it is currently known.
\end{abstract}

Keywords: carcinoma hepatocellular, ablation, radiofrequency, microsensors, microactuator, nanoparticles
Volume 3 Issue I - 2017

\author{
Melissa Silva Monteiro, Guilherme Dos \\ Anjos Guimarães, Bruno Da Costa Motta, \\ Ronei Delfino Da Fonseca, Marina Pinheiro \\ Marques, Gabriel Williams Silva De \\ Mendonça, Suélia Rodrigues Fleury Rosa \\ University of Brasilia, Brazil
}

Correspondence: Melissa S Monteiro, Biomedical Engineering Laboratory (LaB), University of Brasilia/Brazil, Condomínio Ecológico Parque Do Mirante, Rua D, Casa 97, Brasília, Brazil, Tel +5561981300249, Email melcomenator@gmail.com

Received: July 31, 2017| Published: September 13, 2017

\section{Abbreviations: RFA, radiofrequency ablation}

\section{Introduction}

The World Health Organization ranks liver cancer among the five cancers with the highest death rate in the world. ${ }^{1}$ Currently, in the treatment of this disease are practiced, for example, resection of hepatic tissue and radiofrequency ablation, a non-invasive method. Although the resection presents the best results, only $10 \%$ to $15 \%$ of those affected by this disease can perform this procedure. ${ }^{2}$ For radiofrequency ablation (RFA), which consists of the burning of the tumor tissue from the passage of high current density, treatment occurs in cases of primary hepatic carcinoma with a diameter of up to $3 \mathrm{~cm}$. The radiofrequency ablation process acts by causing local cell necrosis to reach a temperature above $60^{\circ} \mathrm{C}$ and below $100^{\circ} \mathrm{C} .{ }^{3,4}$ The RFA presents advantageous aspects such it does not require the hospitalization of the patient and anesthesia. However, it is necessary to improve fundamental points to increase the effectiveness of this procedure..$^{5}$ As a main point, one can cite the minimization of the attribution of the success of the procedure to the skill and/ or experience of the clinical professional of the same, providing parameters (eg tissue temperature, applied power and tissue impedance) from the radiofrequency equipment itself, optimizing the actuation protocol and the use of imaging equipment used to guide the insertion of the electrode for ablation. In addition, other points such as the uniformity and increase of the volume of ablation are also of high relevance in this field of study.
Great progress was achieved in this area with the introduction of modified electrodes and saline solutions in the ablation region, which allowed a significant increase in necrosis volume using a single electrode. ${ }^{6}$ Other advances were also obtained in the monitoring and analysis of the RFA procedure, with the application of fiber optic sensors to perform the online monitoring of the temperature in the region of ablation. ${ }^{7}$ The temperature analysis is able to quantify the heat distribution in the ablation region and thereby determine the efficiency of the procedure performed. ${ }^{8}$ In this work, the next section will discuss the challenges and proposals for some of the complexities to be overcome in the ablation by RFA, followed by a brief conclusion on the subject by the authors of this article.

\section{Discussion}

Necrosis volumes limited to $3 \mathrm{~cm}$ can be explained by factors related to RFA. Current generators can be used with powers between $50 \mathrm{~W}$ and $200 \mathrm{~W}$ with control algorithms that include temperature or impedance in the tissue region. However, studies show that power is not the only determining factor in obtaining larger volumes. ${ }^{9}$ Temperature or impedance control allows better results and can be optimized. Control algorithms that use temperature as a parameter must include losses due to blood perfusion that acts as a heat sink minimizing the effects of temperature propagation. ${ }^{10}$ This temperature should be guaranteed throughout the tumor volume to avoid recurrence in regions that did not reach this temperature. The possibility of reducing the size of the sensors and circuits using, for example, 
MEMS (Micro-Electro Mechanical Systems) type components consisting of integrated devices in the order of a few micrometers up to a few millimeters may comprise a complete microelectronic system with microsensor and microactuators. ${ }^{11}$ The use of MEMS would allow the insertion of microcomponents into the actual volume of the tumor. Thus, parameters such as point temperature, impedance and positioning of the electrode can be acquired more accurately and give greater accuracy to the power control algorithms of the ablation equipment and the adequacy of the electrode geometry to the area of necrosis.

Also in the application of technologies with microcomponents, it is possible to propose a microsystem for the insertion of fluids in the tissue during the ablation process. The fluids can act to accelerate the ablation process (greater volume of burning in less time) or to cool the ablation site (delay the arrival at the carbonization temperature). In the work of Evans et al., ${ }^{12}$ a mixed microsystem for drug infusion is proposed in patients with chronic pain, using two pressurized chambers with springs controlled by two independent micro valves and a microcontroller, regulating the flow and presenting satisfactory efficiency In the delivery of the fluid. Despite the application in another focus of Biomedical Engineering, the method is useful as an initial understanding for use during the radiofrequency ablation process. In the field of nanobiotechnology, the use of metallic magnetic nanoparticles to induce local hyperthermia in tumor tissues through radiofrequency interaction has been shown to be a potentially more effective therapeutic option by improving the transfer of temperature in the ablation area and in a controlled manner. ${ }^{13}$ The use of chemotherapeutic agents, such as gemcitabine, conjugated to gold nanoparticles during ablation by radiofrequency in cases of hepatocellular carcinoma allows to reduce the dose and to apply it locally eliminating some disadvantages of its systemic application in the patient during its recovery. ${ }^{14}$ Increased necrosis area has been demonstrated with the association of iron oxide $(\mathrm{FeO})$ nanoparticles in cases of cardiac arrhythmia due to changes in thermal and electrical conductivity caused by large changes in tissue impedance..$^{15}$ Finally, the perspectives are increasing considering the advance of the lines of research with nanoparticles as propeller of the benefits of the use of RFA.

\section{Conclusion}

The paradigm that involves the state of the art of RFA induces the continuity of research in view of the various advantages of RFA such as low invasiveness, hospitalization and anesthesia unnecessary, as well as a good survival to its patients. There is research with sufficient evidence to redirect this concept to the new technological projections that will make it more effective in its ablation process, generating enough volumetric necrosis for complete regression of the tumor added to a high survival rate. In addition to the adjustments already in progress to use better images to guide the health professional during electrode positioning and combined use with other therapies, new technologies such as microsensors and microactuators, magnetic metallic nanoparticles and more mathematical models which can possibility of RFA in the treatment of tumor nodules and the increase of its effectiveness. The new challenges present themselves as possibilities and reshape the concept of the use of RFA already established.

\section{Acknowledgements}

The research group thanks the federal entities that supported the project: Ministry of Health of Brazil throught the Department of the Industrial Complex and Innovation in Health (DECIIS), Center for Technological Development (CDT / UnB), National Council for Scientific and Technological Development (CNPq) And Coordination of Improvement of Higher Level Personnel (Capes).

\section{Conflict of interest}

The author declares no conflict of interest.

\section{References}

1. Siegel R, Naishadham D, Jemal A. Cancer statistics, 2012. CA Cancer J Clin. 2012;62(1):10-29.

2. Ren H, Enrique Campos-Nanez, Ziv Yaniv, et al. Treatment planning and image guidance for radiofrequency ablation of large tumors. IEEE Journal of Biomedical and Health Informatics. 2014;18(3):920-928.

3. Marcelo Augusto Fontenelle Ribeiro Jr, Renata Potonyacz Colaneri, Bárbara Dos Santos Nunes, et al. Ablação por radiofrequência de tumores hepáticos primários e metastáticos: experiência em 113 casos. Arquivos Brasileiros de Cirurgia Digestiva. 2006;20:38-40.

4. Marques MP. Desenvolvimento de um eletrodo expansível de niqueltitânio para ablação hepática por radiofrequência. Dissertação de Mestrado em Eng Biomédica, Brazil: Universidade de Brasília; 2017.

5. Germani G, Maria Pleguezuelo, Tim Meyer, et al Clinical outcomes of radiofrequency ablation, percutaneous alcohol and acetic acid injection for hepatocelullar carcinoma: a meta-analysis. Journal of hepatology. 2010;52(3):380-388

6. Lencioni R, Cioni D, Crocetti L, et al. Percutaneous ablation of hepatocellular carcinoma: state-of-the-art. Liver Transplantation. 2004;10(S2):S91-S97.

7. Daniele Tosi, Edoardo Gino Macchi, Giovanni Braschi, et al. Fiberoptic combined FPI/FBG sensors for monitoring of radiofrequency thermal ablation of liver tumors: ex vivo experiments. Applied Optics. 2014;53(10):2136-2144.

8. Daniele Tosi, Edoardo Gino Macchi, Mario Gallati, et al. Fiber-optic chirped FBG for distributed thermal monitoring of ex-vivo radiofrequency ablation of liver. Biomedical Optics Express. 2014;5(6):1799.

9. John P Mc Gahan, Shaun Loh, Fernando J Boschini, et al. Maximizing parameters for tissue ablation by using an internally cooled electrode. Radiology. 2010;256(2):397-405.

10. Enrique J Berjano. Theoretical modeling for radiofrequency ablation: state-of-the-art and challenges for the future. Biomedical engineering online. $2006 ; 5: 24$

11. Prime Faraday Partnership, an Introduction to MEMS. Wolfson school of mechanical and manufacturing engineering. England: Loughborough University; 2012.

12. Evans AT, Park JM, Chiravuri S, et al. Dual drug delivery device for chronic pain management using micromachined elastic metal structures and silicon microvalves. IEEE/ASME international conference on micro electro mechanical systems (MEMS 08); USA: IEEE; 2008. p. 252-255.

13. Kim KS, Hernandez D, Lee SY. Time-multiplexed two-channel capacitive radiofrequency hyperthermia with nanoparticle mediation. Biomedical engineering online. 2015;14 95.

14. Raoof M, Warna D Kaluarachchi MS, Sophia Phounsavath, et al. Gold nanoparticles and radiofrequency in experimental models for hepatocellular carcinoma. Nanomedicine: Nanotechnology, Biology and Medicine. 2014;10(6):1121-1130.

15. Duy $\mathrm{T}$ Nguyen, Wendy $\mathrm{S}$ Tzou, Lijun Zheng, et. al. Enhanced radiofrequency ablation with magnetically directed metallic nanoparticles. Circulation: Arrhythmia and Electrophysiology. 2016;9(5):e003820. 